Ann. Zootech., I965, 14 (2), I8I-I89.

\title{
RELATION ENTRE LES DÉPÓTS ADIPEUX VISCÉRAUX ET LES LIPIDES CORPORELS CHEZ LE POULET
}

\author{
P. DELPECH et F. H. RICARD \\ Station de Recherches avicoles, \\ Centre national de Recherches zootechniques, Jouy-en-Josas (Seine-et-Oise), \\ Station expérimentale d'Aviculture du Magneraud, \\ Saint-Pierre-d'Amilly (Charente Maritime)
}

\section{SOMMAIRE}

Nous avons pu démontrer l'existence d'une relation étroite entre le poids des lipides corporels et le poids des tissus adipeux viscéraux chez le coquelet entre 8 et I 9 semaines.

Nos résultats sont discutés en fonction de la composition du régime. Ils montrent que la relation n'est pas la même selon le niveau énergétique alimentaire. Il est donc indispensable de bien contrôler les conditions d'élevage si l'on veut appliquer convenablement la méthode.

On mesure encore fréquemment l'efficacité alimentaire par le gain de poids des animaux. De même, les études génétiques de croissance visent généralement l'amélioration pondérale globale des individus. Or les variations de poids vif concordent rarement avec un développement harmonieux des différents tissus. En effet une augmentation des tissus adipeux peut souvent représenter la majeure partie du gain de poids. La détermination de l'importance de ces dépôts revêt donc un grand intérêt.

Pour l'apprécier chez les mammifères domestiques on a recours à la dissection complète de préférence à l'extraction des lipides corporels. Ces opérations n'en demeurent pas moins longues et délicates. Aussi de nombreuses méthodes d'estimation indirecte ont été mises en ouvre. Elles correspondent à des appréciations subjectives, à des mesures physiques ou chimiques. Leur description a été passée en revue par Dumont, 1958 .

Chez les oiseaux l'extraction des lipides est plus aisée que chez les mammifères. Elle est d'ailleurs plus souvent pratiquée que la dissection totale. Mais le contrôle d'un grand nombre d'individus en limite l'usage pour les études génétiques. Ici encore des méthodes indirectes ont été éprouvées faisant appel à l'appréciation subjective de la répartition des dépôts adipeux sur la carcasse (Combs et HébackA, r959), à 
l'analyse chimique d'échantillons de muscles et de la peau de la cuisse (MEHNER et ToRges, I962) sans contrôler la validité de leur emploi.

Nous avons voulu examiner si les tissus adipeux de la cavité abdominale, quoiqu'ils contiennent de l'eau et du conjonctif en plus des lipides, représentent d'une manière convenable l'ensemble des lipides corporels.

\section{MATÉRIEL E'T MÉTHODES}

Nous utilisons comme matériel expérimental des poussins mâles issus du croisement commercial TCA I 17 de type chair. Ces oiseaux sont répartis en 2 lots, élevés au sol et éclairés 12 heures par jour. Ils reçoivent deux régimes à base de maïs et de tourteau de soja cuit. Ceux-ci renferment i9 p. 100 de matières azotées totales et 2630 calories métabolisables pour l'un et $345^{\circ}$ pour l'autre. Ce supplément d'énergie est apporté par le remplacement de Io p. I00 de cellulose et de I p. Ioo d'amidon par I I p. roo d'huile de mais.

A $8,10,12,14$ et I9 semaines, 9 animaux de chaque régime sont sacrifiés par asphyxie puis pesés. Ils sont choisis au hasard parmi les poulets dont le poids vif est voisin de la moyenne du groupe auquel ils appartiennent.

Après réfrigération des animaux entiers, nous prélevons et pesons les dépôts adipeux viscéraux, constitués par le tissu adipeux mésentérique qui comprend aussi le dépôt qui entoure le gésier. Ils sont ensuite réincorporés dans la carcasse. Celle-ci est alors broyée et une partie aliquote est lyophylisée. Les lipides sont extraits par la méthode de KUMAGAWA : 8 heures à l'alcool éthylique à 95 p. Ioo et 2 heures à l'éther éthylique. L'extrait est desséché puis repris par le benzène. Nous appelons lipides totaux l'extrait ainsi obtenu.

\section{RÉSULTATS}

L'évolution des lipides totaux et du tissu adipeux viscéral est rapportée dans les tableaux I et 2 .

Pour tous les prélèvements il existe une grande variabilité des valeurs des lipides totaux et des dépôts adipeux viscéraux. Elle est soulignée par l'importance des écarts-type. Son amplitude est d'ailleurs plus marquée pour les tissus abdominaux

TABLEAU I

Evolution des lipides totaux corporels et du tissu adipeux viscéral (valeurs en $g$ )

Régime de base

\begin{tabular}{|c|c|c|c|c|c|}
\hline Age en semaines & 8 & 10 & 12 & $1{ }^{\prime} t$ & 19 \\
\hline Poids mort des poulets & $\begin{array}{r}9_{1}^{\prime} 1 \\
+\quad 21(1)\end{array}$ & $\begin{array}{r}1345 \\
\ldots \quad 20\end{array}$ & $\begin{array}{r}1865 \\
=\quad 13\end{array}$ & $\begin{array}{r}2321 \\
\pm \quad 32\end{array}$ & $\begin{array}{r}3149 \\
\pm \quad 14\end{array}$ \\
\hline Lipides totaux corporels & $\begin{aligned} & 60 \\
\therefore \quad & 13,2\end{aligned}$ & $\begin{array}{r}92 \\
\pm \quad 16,1\end{array}$ & $\begin{array}{c}135 \\
+\quad 23,7\end{array}$ & $\begin{array}{c}186 \\
\pm \quad 23,9\end{array}$ & $\begin{array}{r}237 \\
+\quad 58,0\end{array}$ \\
\hline Tissu adipeux viscéral & $\begin{array}{r}3,0 \\
\pm \quad 2,7\end{array}$ & $\begin{array}{r}4,7 \\
\therefore \quad 2,2\end{array}$ & $\begin{array}{r}6,8 \\
+\quad 2,7\end{array}$ & $\begin{array}{r}9,6 \\
\pm \quad 4,8\end{array}$ & $\begin{array}{r}11,9 \\
+\quad 8,0\end{array}$ \\
\hline
\end{tabular}


TABLEAU I (Suite)

Régime de base + huile de maïs

\begin{tabular}{|c|c|c|c|c|c|}
\hline Poids mort des poulets & $\begin{array}{r}857 \\
+\quad 28\end{array}$ & $\begin{array}{r}1403 \\
+\quad 15\end{array}$ & $\begin{array}{r}1736 \\
+\quad 21\end{array}$ & $\begin{array}{r}2191 \\
\div \quad 71\end{array}$ & $\begin{array}{r}2980 \\
\pm \quad 31\end{array}$ \\
\hline Lipides totaux corporels & $\begin{array}{ll} & 83 \\
\pm & 19,1\end{array}$ & $\begin{array}{c}144 \\
-\quad 35,8\end{array}$ & $\begin{aligned} & 179 \\
\quad & 21,0\end{aligned}$ & $\begin{aligned} & 249 \\
-\quad & 36,1\end{aligned}$ & $\begin{array}{r}342 \\
+\quad 79,8\end{array}$ \\
\hline Tissu adipeux viscéral & $\begin{array}{r}6,5 \\
-\quad 4,5\end{array}$ & $\begin{array}{r}12,2 \\
+\quad 10,8\end{array}$ & $\begin{array}{r}11,1 \\
\div \quad 4,9\end{array}$ & $\begin{array}{r}25,4 \\
+\quad 8,5\end{array}$ & $\begin{array}{r}34,2 \\
+\quad 22,8\end{array}$ \\
\hline
\end{tabular}

(1) Écart-type

que pour les lipides corporels. Ainsi dans un même lot un poulet possède 0,08 p. roo de tissu viscéral tandis qu'un autre en renferme 0,88 p. Ioo. La teneur en lipides varie dans les mêmes conditions de 5,43 à Io, 55 p. Ioo. Cette variabilité attire d'autant plus l'attention que nous avons choisi des animaux de poids voisin à chaque âge.

TABLEAU 2

Évolution des lipides totaux corporels et du tissu adipeux viscéral (valeurs relatives)

Régime de base

\begin{tabular}{|c|c|c|c|c|c|}
\hline Age en semaines & 8 & 10 & 12 & $11^{\prime}$ & 19 \\
\hline $\begin{array}{l}\text { Lipides totaux corporels } \\
\% \text { du poids mort }\end{array}$ & $\begin{array}{ll} & 6,41 \\
\pm \quad & 1,49(1)\end{array}$ & $\begin{array}{r}6,8 j \\
-:-1,14\end{array}$ & $\begin{array}{r}7,23 \\
\pm 1,25\end{array}$ & $\begin{array}{r}8,04 \\
+1,14\end{array}$ & $\begin{array}{r}7,55 \\
\pm 1,82\end{array}$ \\
\hline $\begin{array}{l}\text { Tissus adipeux viscéral } \\
\% \text { du poids mort }\end{array}$ & $\begin{array}{r}0,320 \\
\therefore \quad 0,266\end{array}$ & $\begin{array}{r}0,352 \\
-1.0,161\end{array}$ & $\begin{array}{r}0,366 \\
+\quad 0,143\end{array}$ & $\begin{array}{r}0,416 \\
+\quad 0,216\end{array}$ & $\begin{array}{r}0,377 \\
+0,254\end{array}$ \\
\hline
\end{tabular}

Régime de base + huile de Mais

\begin{tabular}{|c|c|c|c|c|c|}
\hline $\begin{array}{l}\text { Lipides totaux corporels } \\
\% \text { du poids mort }\end{array}$ & $\begin{array}{r}9,37 \\
\pm 2,29\end{array}$ & $\begin{array}{r}10,24 \\
\pm \quad 2,59\end{array}$ & $\begin{array}{r}10,31 \\
+\quad 1,15\end{array}$ & $\begin{array}{r}11,42 \\
+\quad 2,00\end{array}$ & $\begin{array}{r}11,49 \\
+\quad 2,70\end{array}$ \\
\hline $\begin{array}{l}\text { Tissu adipeux viscéral } \\
\% \text { du poids mort }\end{array}$ & $\begin{array}{r}0,731 \\
+\quad 0,533\end{array}$ & $\begin{array}{r}0,872 \\
+\quad 0,770\end{array}$ & $\begin{array}{r}0,811 \\
\div \quad 0,285\end{array}$ & $\begin{array}{r}1,157 \\
+\quad 0,485\end{array}$ & $\begin{array}{r}1,148 \\
+\quad 0,767\end{array}$ \\
\hline
\end{tabular}

(') Écart-type.

Les valeurs moyennes nous montrent une augmentation, avec l'âge, des dépôts adipeux viscéraux et des lipides corporels. S'il existe une diminution de la teneur en lipides totaux entre I4 et I9 semaines chez les oiseaux qui consomment le régime de base, elle n'est pas significative. Par ailleurs nous notons à tous les âges un dévelop- 
pement plus important des tissus adipeux et des lipides corporels chez les animaux qui reçoivent la ration additionnée d'huile. Nous constatons en outre que la part des dépôts viscéraux est plus grande avec le régime gras (tabl. 3 ).

\section{TABLEAU 3}

Part des tissus adipeux viscéraux dans les lipides corporels en pourcentage.

\begin{tabular}{|c|c|c|c|c|c|}
\hline Age en semaine & 8 & 10 & 12 & 14 & 19 \\
\hline Régime de base & $\begin{aligned} & 4,5 \\
= & 2,6(1)\end{aligned}$ & $\begin{array}{r}5.0 \\
\pm \quad 1,4\end{array}$ & $\begin{array}{r}4,9 \\
+\quad 1,3\end{array}$ & $\begin{array}{r}5,0 \\
+1,9\end{array}$ & $\begin{array}{r}4,6 \\
+\quad 2,2\end{array}$ \\
\hline Règime enrichi en huile & $\begin{array}{r}7,1 \\
\therefore-3,3\end{array}$ & $\begin{array}{r}7,6 \\
+\quad 3.9\end{array}$ & $\begin{array}{r}7,8 \\
\pm \quad 2,5\end{array}$ & $\begin{array}{r}9,8 \\
+\quad 2,9\end{array}$ & $\begin{array}{r}9,1 \\
+\quad 4,1\end{array}$ \\
\hline
\end{tabular}

(1) Écart type

Nous observons peu de variations de cette proportion en fonction de l'âge chez les animaux qui reçoivent le régime de base tandis qu'elle passe de 7,8 à 9,8 p. roo entre $\mathrm{I} 2$ et $\mathrm{I} 4$ semaines chez les oiseaux qui ingèrent la ration la plus énergétique.

Nous avons calculé pour chaque âge et pour l'ensemble des prélèvements les coefficients de corrélation et de régression entre les lipides totaux et les dépôts adipeux viscéraux. Ils sont rassemblés dans le tableau 4 .

TABLEAU 4

Coefficients de corrélation et de régréssion entre lipides totaux et tissu adipeux viscéral (valeurs en $\mathrm{g}$ )

\begin{tabular}{|c|c|c|c|c|c|c|}
\hline \multicolumn{7}{|c|}{ Coefficients de corrélation } \\
\hline Age en semaines & 8 & 10 & 12 & 14 & 19 & Tous âges \\
\hline Régime de base & $+0,92$ & $+0,92$ & $+0,90$ & $+0,77$ & $+0,87$ & $+\quad 0,82$ \\
\hline Règime de base + Huile de maïs & $+0,92$ & $+0,9^{\prime}$ & $+0,,^{\prime} 3\left({ }^{2}\right)$ & $+0,87$ & $+0,95$ & $+\quad 0,88$ \\
\hline
\end{tabular}

Coefficients de regression

\begin{tabular}{|c|c|c|c|c|c|c|}
\hline Règime de base & $\begin{array}{l}+\quad 4,89 \\
\pm \quad 0,78(1)\end{array}$ & $\begin{array}{l}+0,76 \\
\pm 1,07\end{array}$ & $\begin{array}{l}+\quad 7,92 \\
\pm 1,44\end{array}$ & $\begin{array}{l}+\quad 3,81 \\
\pm 1,18\end{array}$ & $\begin{array}{l}+6,34 \\
\pm 1,31\end{array}$ & $\begin{array}{l}+10,45 \\
\pm \quad 1,10\end{array}$ \\
\hline Règime de base + Huile de maiss & $\begin{array}{l}+\quad 3,84 \\
\pm 0,68\end{array}$ & $\begin{array}{l}+3,11 \\
\pm 0,44\end{array}$ & $+{ }_{(2)}^{1,8^{\prime} t}$ & $\begin{array}{l}+\quad 3,30 \\
\pm 0,83\end{array}$ & $\begin{array}{l}+3,36 \\
\pm 0,40\end{array}$ & $\begin{array}{l}+5,13 \\
\pm \quad 0,52\end{array}$ \\
\hline
\end{tabular}

(1) Écart-type

$\left({ }^{2}\right)$ Non significatif 
Les coefficients de corrélation sont statistiquement significatifs et indiquent une liaison étroite entre ces deux mesures, à une exception près : régime gras à I2 semaines. Il est vraisemblable que cette anomalie est due aux mauvaises conditions d'élevage de ce lot entre Io et I 2 semaines. Nous avons effectué un test d'homogénéité de l'ensemble de ces coefficients (MORICE et Chartier, I954). Ceux-ci peuvent être considérés comme appartenant à une même population (probabilité de $\chi^{2}$ comprise entre 50 et $70 \mathrm{p}$. I00). La valeur pondérée du coefficient de corrélation est égale à à $+0,88$.

La liaison linéaire est admissible ainsi que nous. le montre le test de vraisemblance indiqué par MORICE et CHARTIER, (I954). Nous pouvons donc représenter la relation qui lie les lipides totaux corporels au tissu adipeux viscéral par des droites. Bien entendu nous observons parmi les coefficients de regression la même exception que pour les coefficients de corrélation.

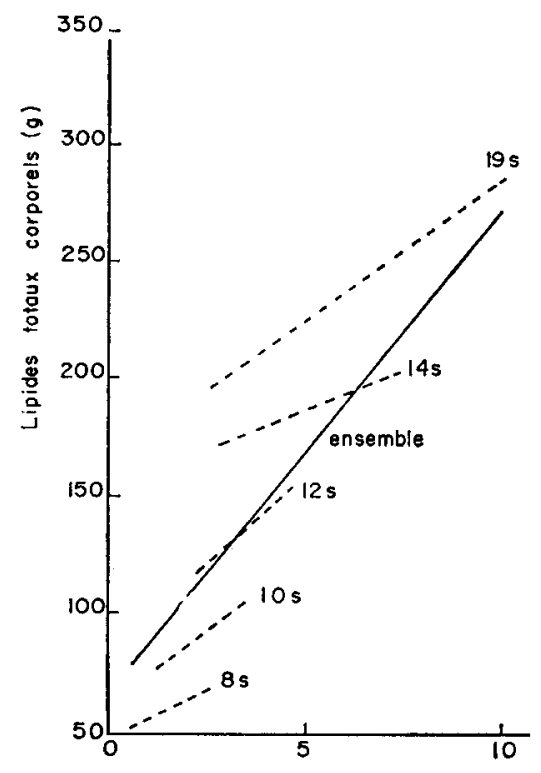

GRPAHIQUE I. - Droites de régression des lipides totaux corporels sur le tissu adipeux viscéral (valeurs exprimées en g) pour le régime de base

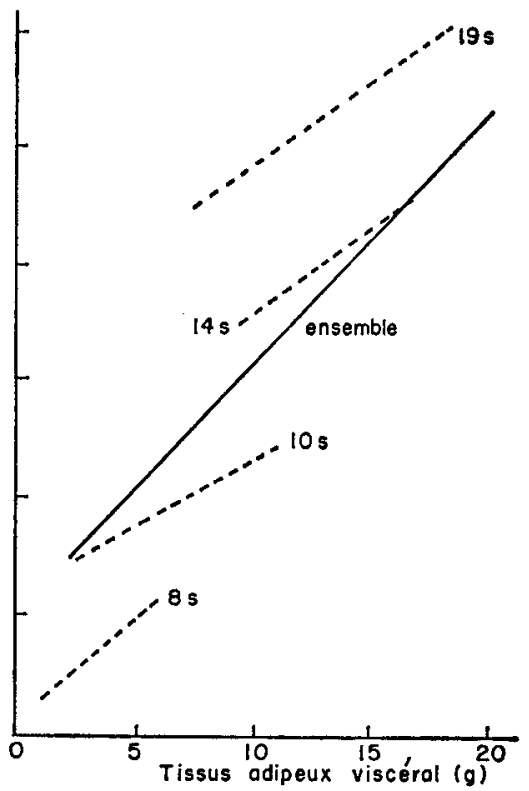

GRAPIIIQte 2. - Droites de régression des lipides totaux corporels sur le tissu adipeux viscéral (valeurs exprimées en g) pour le régime enrichi en huile de mais

(La droite correspondant au prélèvement à i 2 semaines n'a pas de signification ; elle n'a pas été tracée.)

L,es pentes des droites de régression correspondant à l'un ou l'autre des régimes et à un âge donné sont généralement différentes. Par ailleurs, pour un régime donné, le coefficient de régression correspondant à l'ensemble des prélèvements s'écarte notablement de ceux obtenus aux différents âges.

Mais les variances conditionnelles à chaque âge ne sont pas homogènes d'après le test de BARTLET'T (I937). Nous avons pu les homogénéiser en transformant les données et en les exprimant en centièmes du poids mort. 
Dans ces conditions, pour chacun des deux régimes, le test d'homogénéité des pentes, proposé par ULMo, I959 nous indique que la pente définie par la moyenne pondérée des coefficients de régression obtenus à chaque âge ne diffère pas de celle qui

\section{TABLEAU 5}

Coefficients de régression des lipides totaux sur le tissu adipeux viscéral (valeurs réduites à poids constant)

\begin{tabular}{|c|c|c|c|c|c|c|}
\hline Agre en semaines & 8 & 10 & 12 & 14 & 19 & Tous âges \\
\hline Régrime de base & $\begin{array}{l}+1,99 \\
\pm 0,77\left({ }^{1}\right)\end{array}$ & $\begin{array}{l}+6,73 \\
+0,84\end{array}$ & $\begin{array}{l}+7,78 \\
\pm 1,70\end{array}$ & $\begin{array}{l}+4,18 \\
\pm 1,22\end{array}$ & $\begin{array}{l}+6,25 \\
\pm 1,30\end{array}$ & $\begin{array}{l}+5,91 \\
\pm 0,55\end{array}$ \\
\hline $\begin{array}{l}\text { Régime de base }+ \\
\text { Huile de mais }\end{array}$ & $\begin{array}{l}+3,97 \\
\pm \quad 0,63\end{array}$ & $\begin{array}{l}+3,11 \\
\pm 0,45\end{array}$ & $\begin{array}{c}1,86 \\
(2)\end{array}$ & $\begin{array}{l}+3,26 \\
\pm 0,95\end{array}$ & $\begin{array}{l}+3,37 \\
\pm 0,32\end{array}$ & $\begin{array}{l}+3,38 \\
\pm 0,24\end{array}$ \\
\hline
\end{tabular}

(1) Ecart-type

(2) Non significatif

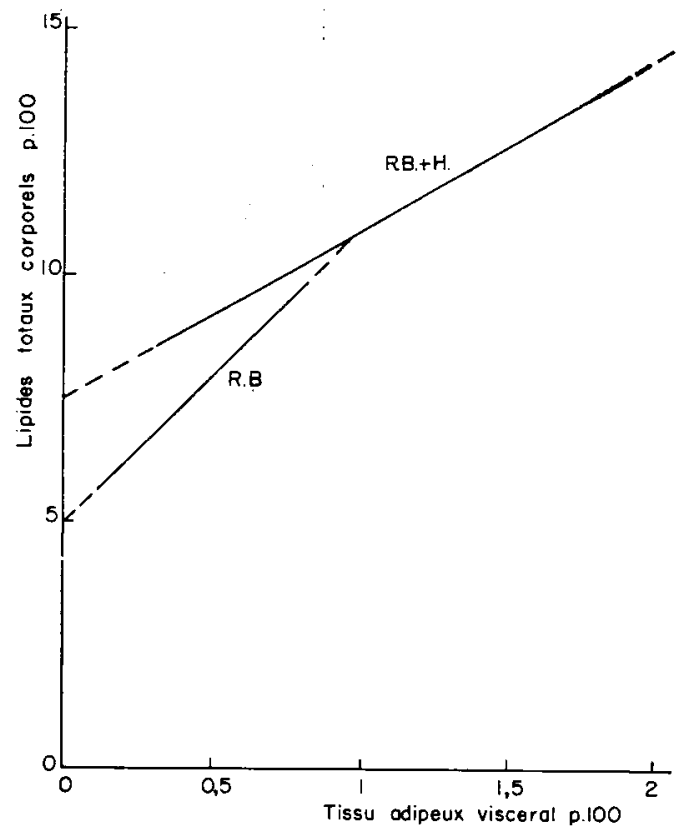

GRAPIIQUE 3. - Droites de régression des lipides tolaux sur le tissu adipeux viscéral correspondanl à l'ensemble des prélèvements (valeurs exprimées en pourcentage du poids mort) R.B. : Régime de base.

R.B. + II : Régime de base enrichi en huile de maïs.

Les limites des traits pleins indiquent les valeurs extrêmes trouvée. 
correspond à l'ensemble des prélèvements. Nous remarquons d'ailleurs que cette dernière pente possède une valeur nettement inférieure à celle que nous obtenons avec les données exprimées en grammes. Par ailleurs les coefficients angulaires des droites représentatives du régime de base et du régime enrichi en huile sont distinctes comme nous l'indique le test $t$ de Student-Fisher. Nous obtenons les équations suivantes :

(Régime de base)

Lipides totaux p. Ioo $=5,9$ tissu adipeux viscéral $p$. IoO $+5,05$.

(Régime enrichi en lipides)

Lipides totaux p. Ioo $=3,4$ tissu adipeux viscéral p. Ioo $+7,28$.

\section{DISCUSSION}

La grande variabilité de la teneur en lipides totaux et de l'importance des dépôts adipeux viscéraux chez les poulets d'un stade donné confirme l'intérêt d'apprécier la composition corporelle des animaux. Il est probable que le comportement alimentaire individuel joue un rôle considérable. Il faudrait étudier cette variabilité en rassemblant des données plus nombreuses qui tiennent compte à la fois du poids des oiseaux et de leurs consommations individuelles.

Nous ne sommes pas les seuls à avoir trouvé une liaison étroite entre les lipides corporels et le tissu adipeux viscéral. POLEY et al. (I940) l'ont déjà indiqué mais ils n'ont pas fourni de justification chiffrée. BOCCARD et DUMONT (I960) ont trouvé une relation analogue chez l'agneau: le coefficient de corrélation entre l'ensemble des tissus adipeux disséqués et le dépôt périrénal est de 0,80 . Cependant la nature de la liaison diffère suivant le mode d'expression des données (valeurs absolues ou valeurs relatives). Dans les graphiques I et 2 les droites obtenues à chaque âge forment une série de marches d'escalier. Il existe donc une discontinuité entre chacun des groupes de mesures. Lorsque nous tentons de les rassembler pour l'ensemble de la période étudiée ( 8 à Ig semaines), nous continuons bien à obtenir une régression linéaire mais la pente de la droite traduit en fait l'augmentation de variance d'un prélèvement à l'autre. Lorsque nous éliminons le poids des animaux de la régression, en comparant les pourcentages de lipides totaux et de dépôts adipeux viscéraux, nous faisons disparaître la discontinuité entre les groupes.

Un autre point remarquable résulte de l'existence d'une ordonnée à l'origine positive des deux droites du graphique 3. Ce fait nous permet de supposer que les dépôts viscéraux apparaissent plus tardivement que la moyenne des autres tissus adipeux. POI,EY et al. (I940) aboutissent déjà à la même conclusion. Ils observent comme nous une proportion plus importante de ces dépôts dans les lipides corporels quand l'âge des animaux passe de $I 7$ à 26 semaines. Ceci correspond à des poids vifs de 1'ordre de $I 200$ et $2500 \mathrm{~g}$ respectivement.

Ces auteurs constatent par ailleurs que, suivant la nature des céréales qu'ils distribuent, la part des lipides des tissus viscéraux dans les lipides corporels varie. LEONG et al. (I959) ont aussi observé une augmentation concomitante des lipides corporels et des dépôts adipeux abdominaux lorsque la ration de poulets de 9 semaines 
est à taux énergétique plus élevé. Nos résultats confirment ces observations. Cela se manifeste par la différence de pente des droites de régression du graphique 3 .

En outre POLEY et al. (I940) enregistrent suivant les régimes une augmentation variable des proportions de tissus adipeux viscéraux dans les lipides corporels, entre I 7 et 26 semaines. Nos résultats concordent également sur ce point. Dans le tableau 3 la proportion de dépôts abdominaux demeure pratiquement constante avec le régime de base. Elle augmente fortement avec la ration enrichie en huile de maiss.

I'élévation du niveau énergétique de la ration accélère la vitesse de formation des tissus adipeux. C'est bien ce que nous montre l'augmentation de la part prise par les dépóts viscéraux dans les lipides totaux chez les poulets qui reçoivent le régime enrichi en énergie. Nous pouvons admettre que cette accélération soit caractéristique de chacun des dépôts pendant une période de croissance donnée. Il s'établirait ainsi une priorité à l'intérieur des tissus adipeux comme il en existe entre les compartiments corporels, selon Hammond (I944).

Reçu pour publication en janvier 1965.

SUMMARY

RELATIONS BETWEEN VISCERAL FAT DEPOSITS AND BOUY LIPIDS IN THE CHICKEN

Cockerels were given two diets based on maize and sova bean, with ig per cent crude protein and differing in that one had i per cent maize oil and the other had none. The diets supplied $345^{\circ}$ and $2630 \mathrm{kcal}$ metabolisable energy per kg. Changes in visceral fat deposits were studied between 8 and 19 weeks in 9 birds of weight representative of each group at each stage.

I. At each age there was a linear relation between the weight of total body lipids obtained by extraction and that of visceral fat deposits or mesenteric fat including that round the gizzard. The slope of the regression for all samples combined differed from those for the separate stages (graphs I and 2). This difference was eliminated when values for the two variables were related to the weight of the birds. There was then a single relation common to all ages (graph. 3).

2. The regressions relating percentage total body lipids and visceral fat deposit differed between the two diets. Abdominal tissue made up a greater proportion of the total lipids in chickens given the diet enriched with oil. That proportion increased with age in those birds. In the birds given the basal diet without oil it remained unchanged (table 3). tissues.

3 . It seemed that the abdominal fat deposit appeared later than the average of the other fatty diet.

4. The relation could be applied in selection as a method for comparing birds fed on the same

\section{RÉFÉRENCES BIBLIOGRAPHIQUES}

Bartlett M. S., 1937. In Morice. M. et Chartier F., r954.

Boccard R., De yonr B. L., ig6o. Note sur la mesure et la variation de l'adiposité des carcasses d'agneau. $V I^{\mathrm{e}}$ Réunion des Instituts de Recherche sur les Viandes, Itrecht, 29 août 3 septembre I960.

Coubs G. F., Helbacka N. Y., 1959. Maryland Broiler Trial. Fat level, animal protein supplements, glycine, pyridoxine, and unextractable soybeans tested with sexes separated. Feedstuffs, 31, $\left(3^{2}\right), 28-34$.

Dumont B. L., I958. Méthodes indirectes de mesure de la graisse corporelles des mammifères. Les Cahiers techniques du Centre national de Coordination des Études et Recherches sur la Nutrition et l'Alimentation, Centre national de la Recherche scientifique, Paris, $195^{\circ}$ 
Hammond J., I944. Physiological factors affecting birth weigth. Proc. Nutr. Soc., 2, 8-14.

Leong K. C., Sunde M. L., Biro H. R., Elvehjey C. A., I959. Interrelationships among dietary energy, protein and amino acids for chickens. Poult. Sci., 38, 1267-1285.

Menner A., Torges If. G., 1962 Methoden zur Feststellungg der Mastfähigkeit und Schlachtwertes bei Hühnern. Arch. Gelügelk., 26, 483-5 I .

Morice E., Chartier F., I954. Méthode Statistique - Deuxième partie - Analyse Statistique. Imprimerie Nationale, Paris, 1954.

Poley W. E., Moxon A. L., Wilson W. O., Dolecek R. L., 1940. Effect of corn, wheat and barley in the diet on the physical and chemical composition of fryers and roasters. J. agric. Res., 61, I6 I-I 78.

Ulmo J., I959. Étude fondamentale de la régression linéaire multiple. Éléments de la théorie des plans d'expérience. Institut de Statistique de l'Université de Paris, I959. 